\title{
Effects of a resistance-training programme on endoplasmic reticulum unfolded protein response and mitochondrial functions in PBMCs from elderly subjects
}

\section{BRISAMAR ESTÉBANEZ ${ }^{1}$, OSVALDO C. MOREIRA ${ }^{1,2}$, MAR ALMAR $^{1}$, JOSÉ A. DE PAZ ${ }^{1}$, JAVIER GONZALEZ-GALLEGO ${ }^{1}$, \& MARÍA J. CUEVAS ${ }^{1}$}

${ }^{1}$ Institute of Biomedicine (IBIOMED), University of León, León, Spain $\mathbb{G}^{2}$ Institute of Biological Sciences and Health, Federal University of Viçosa - Campus Florestal, Florestal, Brazil

\section{Abstract}

Aging has been related with a decline in the ability to handle protein folding, which leads to endoplasmic reticulum stress and alterations in unfolded protein response (UPR). Importantly, physical activity could activate the UPR and attenuate or prevent age-induced ER dysfunction. The current study evaluated the effects of a resistance exercise on UPR and mitochondrial functions in peripheral blood mononuclear cells (PBMCs) from elderly subjects. Thirty healthy women and men (age, 72.8, $s_{x-}=2.2$ years) were randomized to a training group, which performed an 8-week resistance training programme, or a control group, which followed their daily routines. The phosphorylation of PERK and IRE1a, as well as ATF4, and XBP1 protein expression, significantly increased following the training, while expression of BiP, AFT6 and CHOP remain without changes. Additionally, the intervention also induced an increase in PGC-1a and Mfn 1 protein levels, while no changes were found in Drp1 expression. Finally, the resistance protocol was not able to activate PINK1/ Parkin and Bnip3/Nix pathways. The results obtained seem to indicate that 8-week resistance exercise activates the UPR, stimulates mitochondrial biogenesis, maintains mitochondrial dynamics and prevents mitophagy activation by unfolded AQ2 proteins in PBMCs from elderly subjects.

1

Keywords: Aging, endoplasmic reticulum stress, mitophagy, physical activity, strength, unfolded protein response

\section{Introduction}

Aging has been related to loss of proteostasis, which leads to the accumulation of unfolded or misfolded proteins inside the endoplasmic reticulum (ER) lumen. This accumulation triggers ER stress (ERS) and alterations in unfolded protein response (UPR) (Pereira et al., 2016), linked to aging-related diseases (López-Otín, Blasco, Partridge, Serrano, \& Kroemer, 2013). In order to decrease the ER protein load, and thus the ERS, the UPR drives to the upregulation of ER chaperones to promote protein refolding, such as binding immunoglobulin protein $(\mathrm{BiP})$, and to the downregulation of protein translation, through the activation of stress sensors such as protein kinase $\mathrm{R}$ (PKR)-like ER kinase (PERK), inositol-requiring enzyme (IRE) 1 , and activating transcription factor AQ5 (ATF)6 (Naidoo, 2014). Under physiological con1 ditions, the luminal domains of $\mathrm{BiP}$ bind to the three main stress sensors to repress their activity. Nevertheless, unfolded proteins accumulated in the ER lumen dissociate BiP from the effectors PERK, IRE1 and ATF6, which control the expression of downstream transcription factors XBP1 (X-box binding protein 1), ATF4, and ATF6 $\alpha$-p 50, respectively (Nakka, Prakash-Babu, \& Vemuganti, 2016). These factors modulate the expression of proteins involved in redox homeostasis, protein secretion or cell death programmes (Senft \& Ronai, 2015). Moreover, a prolonged ATF4 activation leads to induction of proinflammatory transcription factor C/EBP homologous protein (CHOP) (Nakka et al., 2016).

Aging-associated dysfunction in cell ability to handle protein folding, accumulation and aggregation, may be due to a progressive failure of the chaperoning systems (Naidoo, 2014), as well as to a decline in many of UPR components (Chalil, 
Jaspers, et al., 2015a; Gavilán et al., 2006; Ogborn, McKay, Crane, Parise, \& Tarnopolsky, 2014) causing UPR activation cannot rescue the ERS. Moreover, cellular processes deeply linked to aging, such as autophagy or mitophagy (López-Otín et al., 2013), have been related to UPR activation, evidencing a crosstalk between those pathways (Senft \& Ronai, 2015). Aging affects negatively the immune system, which increases the susceptibility of elderly subjects to infection, autoimmune disease or cancer (Cao et al., 2017). In this context, UPR has now been recognized for its role in immune cell development, differentiation function and survival, and in regulating immune and inflammatory responses, including those associated with infections, tumours and autoimmune responses (Grootjans, Kaser, Kaufman, \& Blumberg, 2016). In fact, PERK directly enhances the cytolytic activity of macrophages, a type of mononuclear cells, and macrophage-mediated clearance of cancer cells while BiP indirectly promotes pro-inflammatory macrophages by shifting tumoral cytokine secretion (Soto-Pantoja et al., 2017). So, an improved understanding of the UPR in immune and inflammatory cells will eventually lead to the development of novel therapeutic strategies to delay aging.

In recent years, physical activity has been proposed as an inductor of the autophagy process, as well as an inhibitor of inflammation-related pathways and immune response in the elderly (Mejías-Peña et al., 2016a, 2016b; Rodriguez-Miguelez et al., 2014, 2015). In particular, resistance training (RT) has been also associated with an improvement of the UPR (Memme, Oliveira, \& Hood, 2016). Nevertheless, there are very few studies in relation to ERS, aging and exercise, and it is not clear the effect of exercise on UPR in old subjects (Baehr et al., 2016; Drummond et al., 2011; Ogborn et al., 2014).

To this background, the aim of the current study was to evaluate whether the main UPR biomarkers are modified in peripheral blood mononuclear cells (PBMCs) from healthy old subjects in response to an 8-week RT. Additionally, we investigated the effects of RT intervention on mitochondrial biogenesis and dynamics, as well as mitophagy. Our hypothesis was that RT would counteract age-related decline of UPR and that this effect would trigger changes in mitochondrial functions.

\section{Material and methods}

\section{Experimental design}

Two different experiments were performed in the 110 present research. To confirm if UPR decline with age, we initially investigated differences in several
UPR biomarkers between young $(n=10)$ and old $(n=14)$ subjects at rest. The main experimental part of the study was carried out exclusively by old participants $(n=30)$ and was completed in 10 weeks. Subjects performed an 8-week RT and blood samples were collected 1-week prior (pre) and after (post) the exercise protocol.

\section{Subjects}

30 active healthy old ( 11 males and 19 females) and 10 healthy young males subjects volunteered to participate in the study. The inclusion criteria specified not to take any medication known to affect the inflammatory status in the six months prior to or during the study. Subjects which had received previous medication for dyslipidaemia, hypertension and diabetes mellitus were also excluded. Moreover, none of the female participants were taking any hormonal treatment, either before or at the time of the study. Participants did not have any experience in $\mathrm{RE}$, and they were asked to maintain their physical activity routines during the study period. For the main experimental part, before any other activity, a medical screening including anthropometric analysis, the physical activity readiness questionnaire (PARQ), a risk factor quiz, blood pressure measurements, and a basal electrocardiogram test was performed in all the elderly participants. Next, old subjects were randomly assigned to a training group (TG; $n=20$ ) or to a control group (CG; $n=10$ ). Subject characteristics are summarized in Table I. Participants from TG followed an 8-week RE, whereas the control group kept their normal daily routines. All volunteers were informed of the objectives and possible risks of the intervention before individual written consent for participation was obtained. The study followed the principles of the Declaration of Helsinki, and the local ethics committee approved all procedures.

\section{Maximal strength assessment}

In the weeks one and 10, and after a standardized 10min warm up on a cycle ergometer (Tunturi F35, Tunturi ${ }^{\circledR}$, Turku, Finland), maximal voluntary isometric contraction test was carried out in a $45^{\circ}$-inclined leg press device (Gervasport, Madrid, Spain) at $110^{\circ}$ knee flexion, and in a biceps curl bench device (Gervasport) at $90^{\circ}$ elbow flexion. Maximal strength was registered by a strain gauge (Globus Ergometer, Globus, Codogne, Italy). After $\sim 30$ min of rest, one repetition maximum test was performed in the same leg-press and biceps curl bench previously described, and in a seated pec 
Table I. Subject characteristics.

\begin{tabular}{llll}
\hline & Youth & \multicolumn{1}{c}{ TG } & CG \\
\hline Age (years) & $22.5 \pm 2.3$ & $73.7 \pm 2.2$ & $73.8 \pm 2.3$ \\
Height $(\mathrm{m})$ & $1.78 \pm 1.8$ & $1.63 \pm 0.1$ & $1.59 \pm 0.1$ \\
Weight $(\mathrm{kg})$ & $79.7 \pm 2.9$ & $73.0 \pm 14.0$ & $72.0 \pm 9.0$ \\
BMI $\left(\mathrm{kg} / \mathrm{m}^{2}\right)$ & $24.6 \pm 2.5$ & $27.5 \pm 0.7$ & $28.5 \pm 0.8$ \\
\hline
\end{tabular}

deck machine (BH Fitness Nevada Pro, BH, Vitoria, Spain).

\section{Resistance exercise training}

Subjects from the TG completed 16-RT sessions over 8 weeks (two sessions per week), with at least $48 \mathrm{~h}$ between sessions. After a 10-min warm-up on a cycle ergometer, participants performed eight different exercises, i.e. leg press, ankle extension, bench press, leg extension, biceps curl, pec deck, high pulley traction, dumbbell lateral lift in the same exercise devices described above. Participants performed three sets of $12-8-12$ repetitions for each exercise. The load of the first four exercises started at $40 \%$ of one repetition maximum (1RM), increasing the load by $5 \%$ each week. For the last four exercises the load was prescribed according to the rating of perceived exertion (with the OMNI-RE scale, scale of 1 to 10) the first week with an intensity of five, the next three weeks the intensity increased to six and the last four to eight (Gearhart, Jr., Lagally, Riechman, Andrews, \& Robertson, 2009). Between each repetition, the participants had two-three minutes of rest, and between each exercise, there were three minutes of rest.

\section{Blood sample preparation}

Venus blood samples $(30 \mathrm{~mL}$ ) were obtained, in the early morning in the fasted state, from the brachiocephalic vein using the EDTA anticoagulant VacutainerTM systems (BD, Franklin Lakes, NJ, USA), 5-6 days before and after the training period. In the case of the young subjects, the blood samples were collected only once at rest. To avoid circadian effects, all samples were collected between 08:00 and 09:00 am. Participants were required to avoid any intense exercise during the previous 5-6 days. No caffeine or alcohol was allowed during this time. Total blood was centrifuged to isolate plasma and PBMCs using density gradient centrifugation on Ficoll separation solution (Biochrom AG, Berlin, Germany). PBMCs were used as representative cells of the immune system, and also chosen in the interest of practicalities and cost, offering a useful alternative for gene expression analyses as a substitute for tissues that are not easily accessible (Fratta Pasini et al., 2018).

\section{Western blot analysis}

Western blots were carried out in PBMCs as previously described (Mejías-Peña et al., 2016a). Antibodies against ATF6 (Ref. ab65838), ATF4 (Ref. ab1371), Bnip3 (Ref. ab109362), Bnip3L/Nix (Ref. ab109414), Grp78/BiP (Ref. ab21685), Mfn1 (Ref. ab104374), Parkin (Ref. ab15954), PINK1 (Ref. ab23707) and pIRE1 (Ref. ab48187) were purchased from Abcam ${ }^{\circledR}$, Cambridge, UK, USA; Drp1 (Ref. 4494S) was purchased from Cell Signaling Technology ${ }^{\circledR}$, Beverly, MA, USA; Gad153/CHOP (Ref. sc7351), PGC-1a (Ref. sc-13067), pPERK (Ref. sc32577) and XBP1 (Ref. sc-7160) were purchased from Santa Cruz Biotechnology, CA, USA; and $\beta$ actin (Ref. A5060) was purchased from SigmaAldrich, St Louis, MO, USA.

\section{Statistical analysis}

Data are presented as mean, standard error of means $\left(s_{x-}\right)$. Post-training values were normalized to pretraining values. Kolmogorov-Smirnov test was used to verify normal data distribution; when data were skewed, log transformation was used. Comparisons between young and old groups were performed by using Student's t test. The effects of exercise on UPR and mitochondrial functions in the elderly were analysed using a two-way analysis of variance (ANOVA) with repeated measures for group (CG and TG) and time (pre and post). Bonferroni analysis was used to compensate for multiple post hoc comparisons. Differences were considered significant when $P<.05$. All statistical analyses were performed using SPSS version 18 (SPSS Inc., Chicago, IL, USA).

\section{Results}

Subjects characteristics

There were no differences between TG and CG in age, height, body weight, and body mass index 
before and after the intervention. All participants from TG showed a $100 \%$ compliance with the RT.

Maximal voluntary isometric contraction in lower limbs $\left(76.0, s_{x-}=5.0\right.$ vs. $\left.87.6, s_{x-}=5.5 \mathrm{~kg} ; P<.05\right)$ and in upper limbs (48.5, $s_{x-}=4.3$ vs. 63.0, $\left.s_{x-}=5.3 \mathrm{~kg} ; P<.05\right)$ increased in TG from pre to post. Similarly, RT also increased $1 \mathrm{RM}$ in lower limbs (71.0, $s_{x-}=4.7$ vs. $\left.88.3, s_{x-}=4.9 \mathrm{~kg} ; P<.05\right)$ and in upper limbs $\left(55.0, s_{x-}=6.9\right.$ vs. $71.3, s_{x-}=4.6 \mathrm{~kg}$; $P<.05)$. CG showed no functional changes between before and after the intervention period.

\section{Effect of aging on the UPR and mitophagy signalling pathways}

To confirm that UPR processes were impaired in elderly subjects, the protein levels of BiP, PERK phosphorylated (pPERK), IRE1 phosphorylated (pIRE1), ATF6 and ATF4, CHOP and XBP1 were compared between young and old subjects at rest. As illustrated in Figure 1 $(\mathrm{a}-\mathrm{c})$, IRE1 phosphorylation and CHOP and XBP1 levels were significantly decreased in PBMCs from the elderly group when compared with youths $(P<.05, P<.03, P<.02$, respectively). However, PERK phosphorylation and BiP, ATF6 and ATF4 levels did not show differences between young and old participants at rest $(P=192$, $P=.990, P=.372$ and $P=.856$, respectively) (Figure $1(\mathrm{~d}-\mathrm{g}))$. In the same line, mitophagy markers, adenovirus E1B $19 \mathrm{kDa}$-interacting protein (Bnip)3, Nip3like protein X (Nix), PTEN-induced putative kinase (PINK)1, Parkin, and light chain (LC) 3 isoforms I (LC3I) and II (LC3II), were compared between young and old subjects at rest. As illustrated in Figure 1(h,i), Bnip3 and Nix were significantly decreased $(P<.02$ and $P<.001$, respectively) in PBMCs from the elderly. However, there were no significant differences in the protein levels in PINK1/Parkin mitophagy pathway between young and old participants $(P=.089$ and $P=.518$, respectively) (Figure $1(j, k)$ ). Upon confirmation of declined IRE1 and the final stage of PERK UPR arms, as well as Bnip3/Nix mitophagy pathway, with age, we proceeded to analyse the effects of RT on the UPR and mitochondrial functions in elderly subjects.

\section{Effect of RT on the UPR signalling pathways}

Protein expression of the three principal UPR pathways was analysed in human aged PBMCs after 8 -week RT or daily routines. RT did not increase BiP expression $(P=.152)$ (Figure 2(a)). Although no changes in ATF6 levels $(P=.114)$ were found
PERK and IRE1 phosphorylation (Figure 2(c,d)) was significantly higher $(P<.01$ and $P<.02$, respectively) after the RT. Regarding ATF4 (Figure 2(e)), a PERK downstream target, RT induced its protein expression $(P<.02)$. RT increased, although nonsignificantly $(P=.053)$, ATF4-induced pro-inflammatory transcription factor CHOP levels (Figure 2 (f)). Finally, as illustrated in Figure 2(g), RT significantly increased the content of XBP1 compared to pre-training values $(P<.02)$.

\section{Effect of RT on mitochondrial biogenesis and dynamics and mitophagy}

To analyse the effects of RT in PBMCs from elderly subjects in the mitochondrial biogenesis and dynamics processes, and its relationship with UPR and mitophagy, the protein concentrations of peroxisome proliferator-activated receptor-gamma coactivator (PGC) $1 \alpha$, dynamin-related protein (Drp) 1 and mitofusin (Mfn) 1 were measured. As illustrated in Figure 3(a), the concentration of PGC- $1 \alpha$ increased significantly in the TG compared to pretraining values $(P<.05)$, suggesting a mitochondrial biogenesis increase. After the RT, we did not find any change (Figure $3(b))$ in Drp1 levels $(P=.517)$, but the results showed a significant increase of $M f n 1$ expression in the TG compared to pre-training values $(P<.04)$ (Figure $3(\mathrm{c})$ ).

To analyse the effects of RT in PBMCs from elderly subjects on mitophagy process, and its relationship with UPR and mitochondrial functions, we measured the protein levels of the two main mitophagy pathways, PTEN-induced putative kinase 1 (PINK1)/Parkin and adenovirus E1B $19 \mathrm{kDa}$-interacting protein 3 (Bnip3)/Nip3-like protein $\mathrm{X}$ (Nix) mitophagy. The current RT did not affect to mitophagy process. Regarding PINK1/Parkin pathway, no change was found in PINK1 and Parkin expression in the TG compared with pre-training values $(P=.714$ and $P=.607$, respectively) (Figure $3(\mathrm{~d}, \mathrm{e}))$. As illustrated in Figure $3(\mathrm{f}, \mathrm{g})$, no training effect was observed in the Bnip3/Nix pathway, $(P$ $=.079$ and $P=.838$, respectively).

\section{Discussion}

The phosphorylation of PERK and IRE1a, as well as ATF4 and XBP1 protein expression, significantly increased following RT in aged PBMCs, while expression of BiP, AFT6 and $\mathrm{CHOP}$ remain unchanged. Additionally, the intervention also induced an increase in PGC-1a and Mfn 1 protein levels, while no changes were found in Drp1 expression. Finally, the RT does not seem to exert 
$\mathbf{A}$

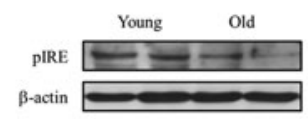

225

230

235

240

245

250

255

G

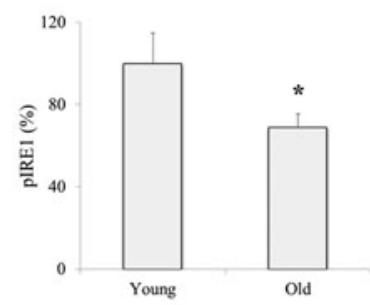

D
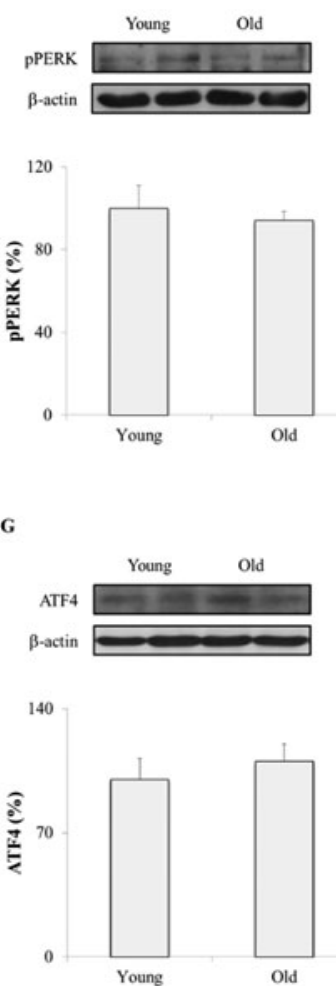

J
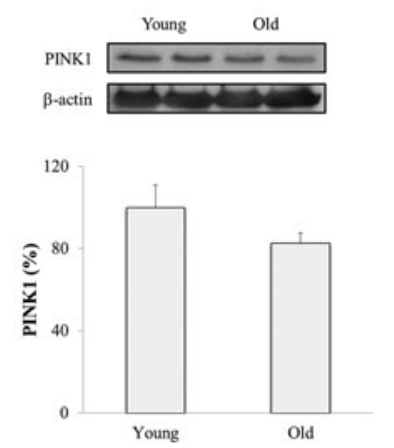

B
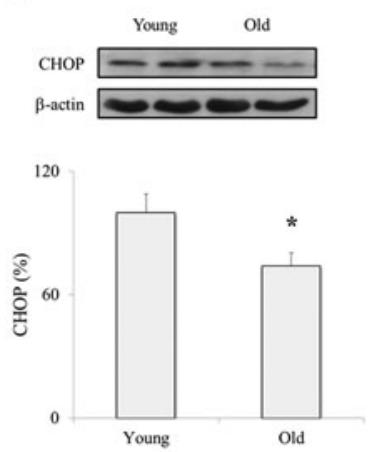

E
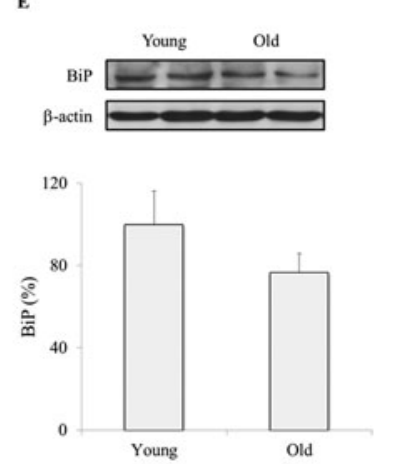

H
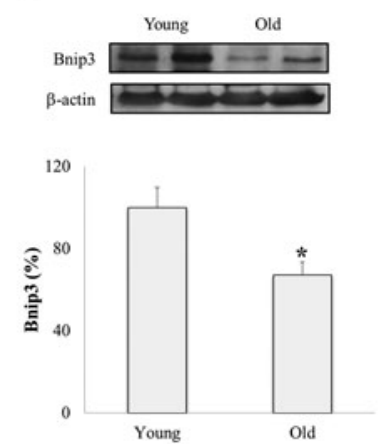

K

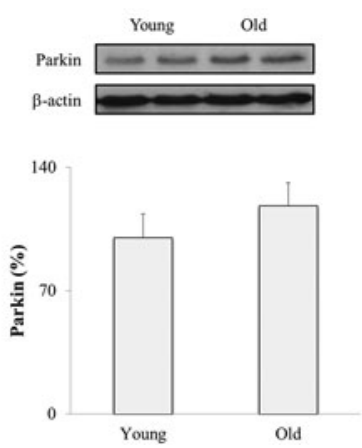

C
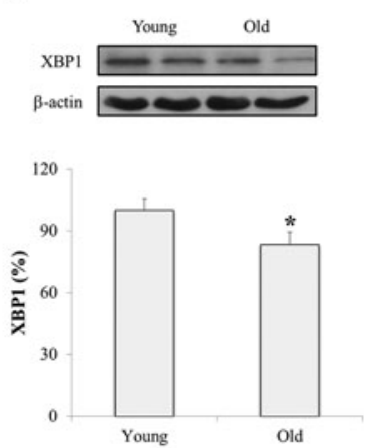

F
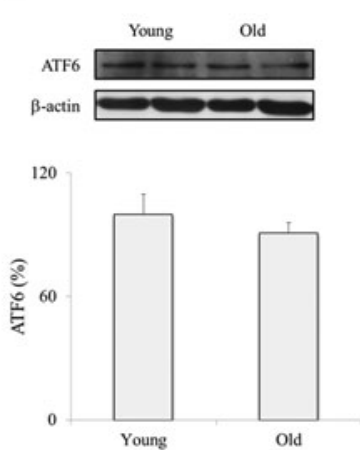

I
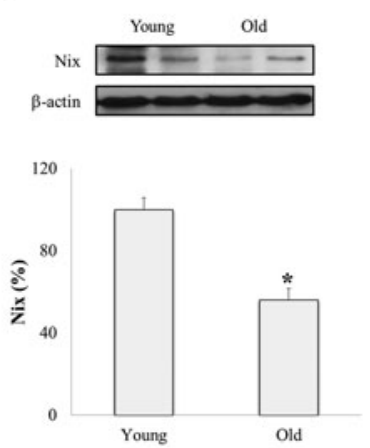

Figure 1. Effects of age on pIRE1, CHOP, XBP1, pPERK, BiP, ATF6, ATF4 Bnip3, Nix, PINK1 and Parkin expression. Densitometric quantification and representative Western blot of pIRE1 (a), CHOP (b), XBP1 (c), pPERK (d), BiP (e), ATF6 (f), ATF4 (g), Bnip3 (h), Nix (i), PINK1 (j) and Parkin (k) in PBMCs from young and old subjects at rest. Protein from PBMCs was separated by sodium dodecyl sulfate-polyacrylamide gel electrophoresis, followed by immunoblotting. Equal loading of proteins is illustrated by $\beta$-actin bands. Values are means, $s_{x-._{i}}{ }^{*} P<.05$ vs. young. 

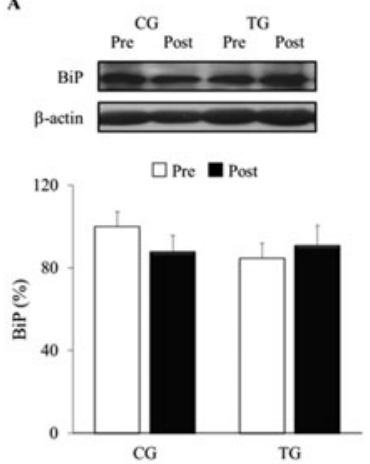

290

295

E
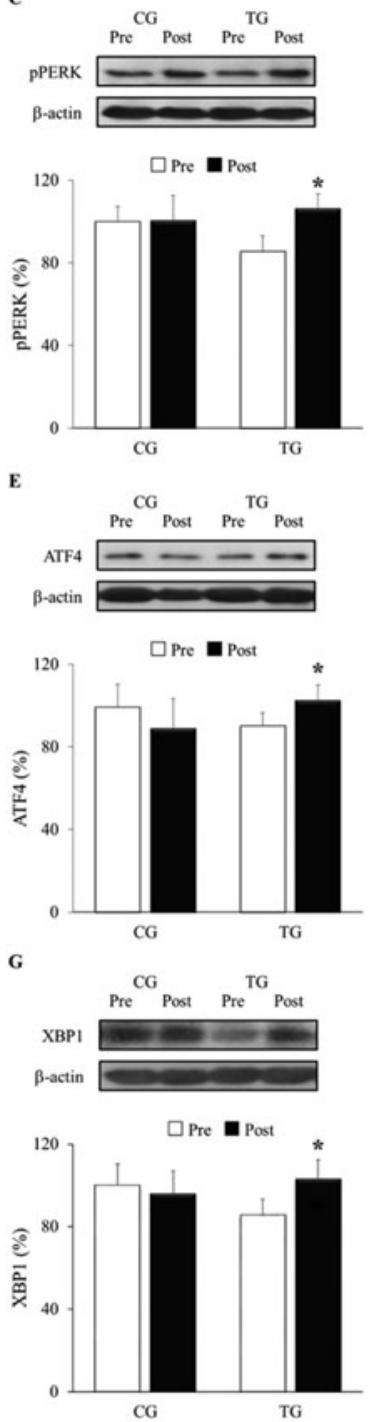

Figure 2. Effects of resistance training on BiP, pPERK, pIRE1, ATF6, ATF4, CHOP and XBP1 expression. Representative Western blots and densitometric quantification of BiP (a), ATF6 (b), pPERK (c), pIRE1 (d), ATF4 (e), CHOP (f) and XBP1 (g) in response to 8-week RT for TG and the same period of normal daily routines for CG. Protein from PBMCs was separated by sodium dodecyl sulfate-polyacrylamide gel electrophoresis, followed by immunoblotting. Equal loading of proteins is illustrated
B
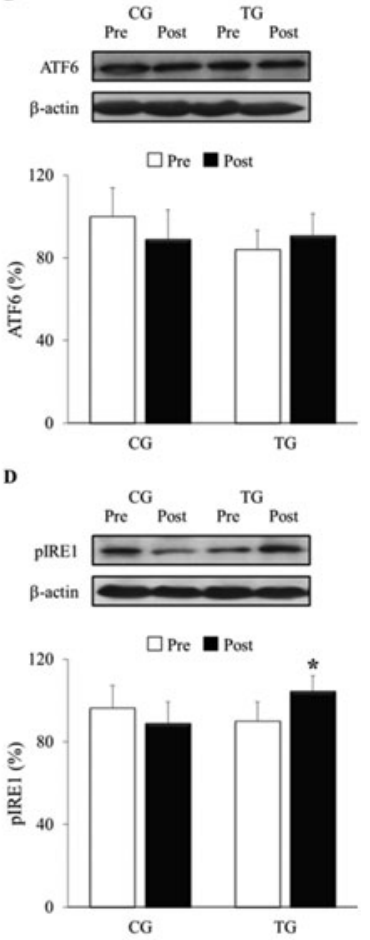

$\mathbf{F}$
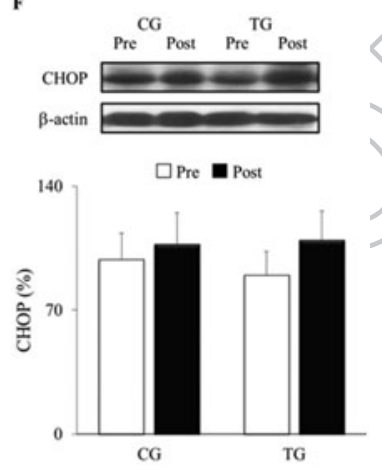
by $\beta$-actin bands. Values are means, $s_{x-.}{ }^{*} P<.05$ vs. Pre within a
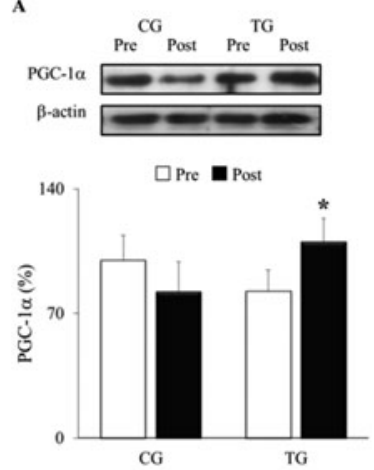

C
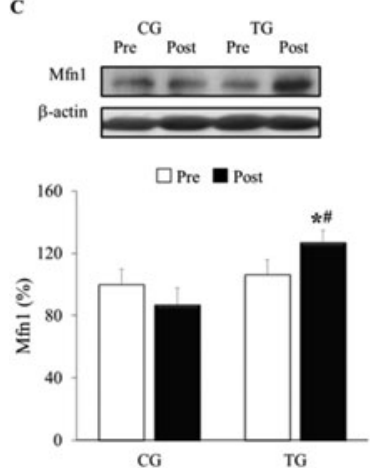

$\mathbf{E}$
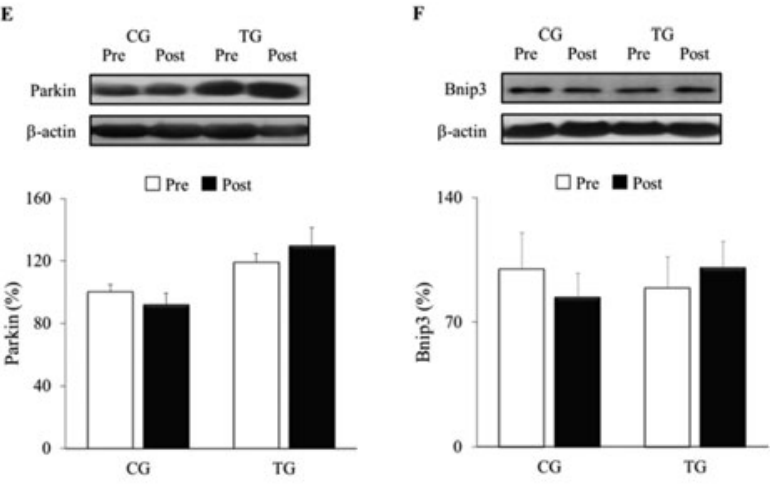

D
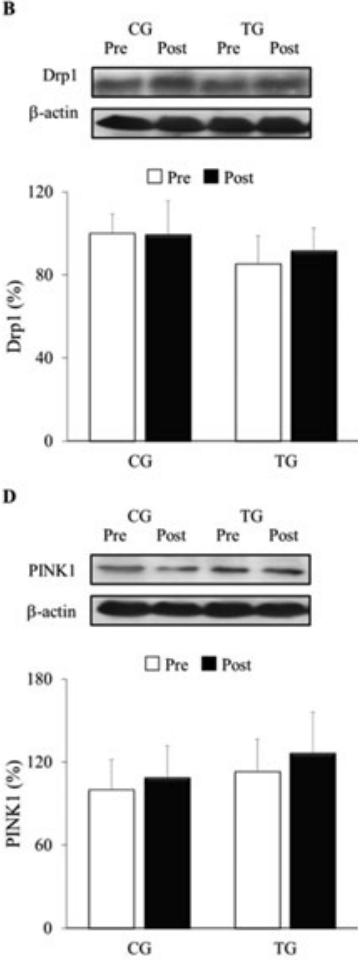

G

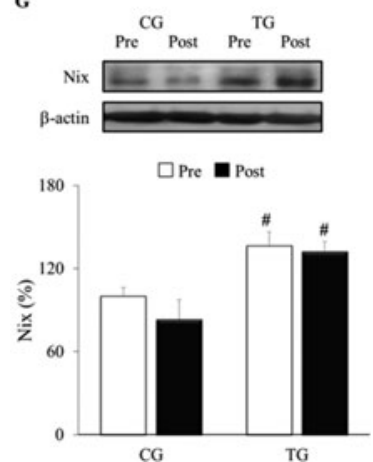
group.

Figure 3. Effects of resistance training on PGC-1 $\alpha$, Drp1, Mfn1, PINK1, Parkin, Bnip3 and Nix expression. Representative Western blots and densitometric quantification of PGC-1 $\alpha$ (a), Drp1 (b), Mfn1 (c), PINK1 (d), Parkin (e), Bnip3 (f) and Nix (g) in response to 8-week RT for TG and the same period of normal daily routines for CG. Protein from PBMCs was separated by sodium dodecyl sulfate-polyacrylamide gel electrophoresis, followed by immunoblotting. Equal loading of proteins is illustrated by $\beta$-actin bands. Values are means, $s_{x-}{ }_{2}{ }^{*} P<.05$ vs. Pre within a group. 
any modification in PINK1/Parkin and Bnip3/Nix pathways.

$\mathrm{BiP}$ is the chaperone that recognizes the unfolded proteins and triggers UPR activation. Our results indicate that BiP levels are not compromised in elderly subjects. In agreement with this, several studies have shown the same results in many tissues, including brain, pancreas kidney or muscle, from both humans and animal models (Baehr et al., 2016; Chalil, Jaspers, et al., 2015; Drummond et al., 2011; Gavilán et al., 2006; Naidoo et al., 2014; Ogborn et al., 2014). However, other authors have described the opposite results depending on the evaluated tissues (Chalil, Jaspers, et al., 2015; Takeda et al., 2013). Moreover, ATF6 has been considered the major transcriptional activator of BiP expression (Borsa et al., 2015) and, as in our case, the few researches that have evaluated ATF6 levels have found the same results for ATF6 as for $\mathrm{BiP}$, which could explain why this protein, involved in the folding process, is not altered with age.

It is important to highlight that the modifications observed in the three pathways in the elderly differ widely among the studies revised. We found a decrease with age in IRE1/XBP1 pathway, as well as in the CHOP final UPR effector, but no changes were observed in pPERK, ATF4 and ATF6. These results contrast with other studies where other 360 authors reported no changes in IRE1 mRNA expression in rat brain and human muscle, respectively (Gavilán et al., 2006; Ogborn et al., 2014), or reported differences even depending in the type of muscle (Chalil, Pierre, et al., 2015; Tamura, Matsunaga, Kitaoka, \& Hatta, 2017). The effects of aging on XBP1 expression are also contradictory including increases, decreases or no changes (Chalil, Jaspers, et al., 2015; Gavilán et al., 2006). On the other hand, CHOP seems to be elevated in most of the mice and rats aged tissues (Chalil, Jaspers, et al., 2015; Tamura et al., 2017). However, Takeda et al. (2013) shown no changes or decreases depending on the tissue analysed. Nevertheless, none of those studies have described the CHOP state on elderly human subjects. In order to explain our results, it should be noted that maximal CHOP expression requires the activation of all three UPR arms (Ogborn et al., 2014) and, in our research, the UPR components show no changes or are decreased. and ATF4 could be an attempt to rescue the ERS in elderly.

Interventional studies have demonstrated that regular exercise is able to decrease oxidative stress and inflammation and reverse mitochondrial and ER dysfunctions (Deldicque, Cani, Delzenne, Baar, \& Francaux, 2013) and could act as a protective mechanism against current and future stressors (Pereira et al., 2016). Our results indicate that 8week RT was able to induce the UPR in PBMCs from elderly subjects. Thus, we found that PERK/ ATF4 and IRE/XBP1 pathways were activated with the protocol. However, unexpectedly, $\mathrm{BiP}$ and ATF6 were not changed with this training. Nevertheless, other studies have found similar results. Thus, BiP expression was not modified in mice soleus, liver and pancreas after 6 -week treadmill (Deldicque et al., 2013). Regarding ATF6, mRNA levels were not changed in rat liver with the 6-week treadmill (Chapados \& Lavoie, 2010). The same results were obtained in ATF6 levels in mice hypothalamus after 8 -week voluntary running (Pinto et al., 2017). This could be explained by the fact that BiP levels depend on those of ATF6 (Borsa et al., 2015), which, in our case, was not increased by exercise. Moreover, components of the UPR have different activation and deactivation timing, depending on the stressor, and ATF6 is involved in an early adaptive response in order to alleviate ERS (Tampakakis et al., 2016). That could explain why a single bout of RT increased BiP and ATF6 mRNA expression at $48 \mathrm{~h}$ in aged human muscle (Ogborn et al., 2014), but chronic training, as for ours, did not (Chapados \& Lavoie, 2010). Another possible reason that may explain the lack of ATF6 changes in our study could be the cooperation of ATF6 with PGC- $1 \alpha$ to regulate $\mathrm{BiP}$ promoter (Wu et al., 2011). In fact, the UPR is modulated by PGC- $1 \alpha$ and ATF6 coactivation (Smiles, Hawley, \& Camera, 2016). The present study demonstrated that PGC1- $\alpha$, the master regulator of mitochondrial biogenesis and UPR as an adaptation to physical activity, increased with our RE. PGC- $1 \alpha$ physically interact with and precipitate the activated form of ATF6 [ATF6 $\alpha(1-$ 373)], but not full-length since ATF6-prior cleavage is needed to form this functional complex (Wu et al., 2011). It is possible that, with the chronic exercise employed, we could not find increases in the ATF6 full-length form measured, since most of this protein could be cleavaged or in complex with PGC- $1 \alpha$.

Regarding the IRE1/XBP1 pathway, we observed activation as shown the increase of pIRE1 and XBP1 levels. Ogborn (2014) found an increased in IRE1 protein and mRNA expression at $24 \mathrm{~h}$ after a single bout of RT in aged human muscle. However, 
6-week treadmill did not modify IRE1 protein and XBP1 mRNA levels in rat liver (Chapados \& Lavoie, 2010) and mice muscle, liver and pancreas (Deldicque et al., 2013). The contradictory results could be due to a different type of exercise, protocols, duration or even different tissue and species. Regarding PERK/ATF4 pathway activation, data showed an increase of pPERK and ATF4 levels, although those results do not match with similar studies. Thus, 12week treadmill decreased pPERK/PERK ratio in mutant presenilin 2 (PS2) mice hippocampus (Kang et al., 2013). However, it is known that IRE1 and PERK activation modulate autophagy, through IRE1-TRAF2-JNK-autophagy axis (H. Wu, Wei, 400 Sehgal, Liu, \& Chen, 2016) or through PERK/ pIF2 $\alpha$ pathway (Deegan, Saveljeva, Gorman, \& Samali, 2013); thereby, the increase observed in pPERK and pIRE1 with RE is in accordance with our previous studies where both aerobic and RT upregulated levels of several autophagy markers (Mejías-Peña et al., 2016a, 2016b).

Furthermore, we found no changes in CHOP levels. Contradictory results have been reported on this protein. Thus, a single bout of RT did not 410 modify CHOP mRNA expression in aged human muscle (Ogborn et al., 2014) whereas 12-week treadmill decreased CHOP protein levels in mutant PS2 mice hippocampus (Kang et al., 2013), and 14-day unloading and reloading protocol increased CHOP levels in aged rats muscle (Baehr et al., 2016). In the present research, an issue to consider is that biological responses vary according to exercise intensity and induce different degrees of ERS and UPR activation (Deegan et al., 2013), and it is important to note that, during prolonged UPR, CHOP leads to apoptosis. In a previous study, we already reported that 8-week RT declined apoptosis, decreasing Bad/ Bcl-2 ratio and caspase-3 levels (Mejías-Peña et al., 2016a). Thus, increased PERK and IRE1 activation and unchanged CHOP could alleviate ERS and inhibit apoptosis.

There is a complex network owing to the crosstalk between UPR and other stress signalling. In fact, ERS contributes to mitochondrial dysfunction,

430 since ER and mitochondria are closely connected, in part, through tethering of these two organelles by the membrane protein mitofusins (Filadi et al., 2015). The RT employed increased Mfn 1 expression, as previously observed in rat skeletal muscle (Kitaoka, Ogasawara, Tamura, Fujita, \& Hatta, 2015), but did not modify Drp1 protein levels. We speculate that the increase in $\mathrm{Mfn} 1$, could improve the ER-mitochondria tethering and signalling, not modifying the overall mitochondrial
The UPR may also modulate mitophagy and take part in identifying weakened mitochondria that need to be eliminated (Chalil, Pierre, et al., 2015; Wu et al., 2016). In the present study, no activation of PINK1/Parkin or Bnip3/Nix pathways post-exercise were found, suggesting that these mitophagy pathways are not stimulated by RT or the UPR could avoid its activation. Until now, there are few studies that have investigated the exercise effects on mitochondrial dynamics or mitophagy specifically, and previous data are not in consensus on the induction of mitophagy with the exercise in elderly subjects (Moreira et al., 2017). Thus, there were no differences in PINK1 mRNA levels in the human muscle between sedentary and active elderly; however, Parkin mRNA expression was increased in active elderly subjects (Drummond et al., 2014). Moreover, 12-week aerobic exercise did not change PINK1 and Parkin mRNA expression in aged human muscle (Fealy, Mulya, Lai, \& Kirwan, 2014) and Bnip3 mRNA expression was increased in muscle from active elderly subjects (Drummond et al., 2014) whereas Bnip3 protein expression was decreased (Zampieri et al., 2015).

In our study, is possible that exercise-induced UPR activation could counteract ERS and lead to mitochondrial improvement, avoiding mitophagy activation. This idea may be supported by the fact that Parkin is upregulated in response to unfolded protein-induced ERS and, if exercise-stimulated UPR can solve the ERS, Parkin levels could not be altered. Finally, we also cannot exclude that the lack of more significant changes in the UPR or in the mitophagy after the RE, could be due to the recruitment of previously physically active old subjects in the study. However, despite this limitation, to our knowledge, this is the first time that UPR and its crosstalk with mitochondrial biogenesis, dynamics and mitophagy is studied in PBMCs from healthy elderly subjects in response to RE.

In summary, despite the limitation in the present study due to the use of only Western blot and the exclusive observation of markers of the evaluated processes, our results show that 8 -week RT induced the $\mathrm{UPR}$, as a protective mechanism, and mitochondrial biogenesis, to deal with the exercise demands, but did not modify mitophagy in PBMCs from elderly population. However, it remains to be elucidated whether UPR activation after 8-week RT is an adaptive response to exercise, which precedes mitochondrial changes in dynamics and mitophagy, or avoids these processes by dealing with the stress caused by unfolded or misfolded proteins. Thereby, data should serve to develop further studies in order to identify the mechanism by which exercise upregulates 
1 UPR and how this is related to mitochondrial functions.

\section{Disclosure statement}

AQ7 AQ6 No potential conflict of interest was reported by the authors.

\section{1}

450

\section{Funding}

This work was supported by O.C. Moreira (Coordenação de AperAQ4 AQ3 feiçoamento de Pessoal de N); B. Estébanez (Ministry of Edu$1 \quad 1$ cation of Spain FPU fellowship).

455

\section{References}

Baehr, L. M., West, D. W., Marcotte, G., Marshall, A. G., De Sousa, L. G., Baar, K., \& Bodine, S. C. (2016). Age-related deficits in skeletal muscle recovery following disuse are associated with neuromuscular junction instability and ER stress, not impaired protein synthesis. Aging (Albany NY), 8(1), 127-146.

Borsa, M., Ferreira, P. L., Petry, A., Ferreira, L. G., Camargo, M. M., Bou-Habib, D. C., \& Pinto, A. R. (2015). HIV infection and antiretroviral therapy lead to unfolded protein response activation. Virology fournal, 12(77).

Cao, D. H., Beyer, I., Mets, T., Onyema, O. O., Njemini, R., Renmans, W., ... Bautmans, I. (2017). Effects of physical exercise on markers of cellular immunosenescence: A systematic review. Calcified Tissue International, 100(2), 193-215.

Chalil, S., Jaspers, R. T., Manders, R. J., Klein-Nulend, J., Bakker, A. D., \& Deldicque, L. (2015). Increased endoplasmic reticulum stress in mouse osteocytes with aging alters Cox-2 response to mechanical stimuli. Calcified Tissue International, 96(2), 123 128.

Chalil, S., Pierre, N., Bakker, A. D., Manders, R. J., Pletsers, A., Francaux, M.,... Deldicque, L. ( $\left.x_{015}\right)$. Aging related ER stress is not responsible for anabolic resistance in mouse skeletal muscle. Biochemical and Biophysical Research Communications, 468(4), 702-707.

Chapados, N. A., \& Lavoie, J. M. (2010). Exercise training increases hepatic endoplasmic reticulum (er) stress protein expression in MTP-inhibited high-fat fed rats. Cell Biochemistry and Function, 28(3), 202-210.

Deegan, S., Saveljeva, S., Gorman, A. M., \& Samali, A. (2013). Stress-induced self-cannibalism: On the regulation of autophagy by endoplasmic reticulum stress. Cellular and Molecular Life Sciences: CMLS, 70(14), 2425-2441.

Deldicque, L., Cani, P. D., Delzenne, N. M., Baar, K., \& Francaux, M. (2013). Endurance training in mice increases the unfolded protein response induced by a high-fat diet. Fournal of Physiology and Biochemistry, 69(2), 215-225.

Drummond, M. J., Addison, O., Brunker, L., Hopkins, P. N., McClain, D. A., LaStayo, P. C., \& Marcus, R. L. (2014). Downregulation of E3 ubiquitin ligases and mitophagy-related genes in skeletal muscle of physically inactive, frail older women: A cross-sectional comparison. The fournals of Gerontology Series A: Biological Sciences and Medical Sciences, 69 (8), 1040-1048.

Drummond, M. J., Fry, C. S., Glynn, E. L., Timmerman, K. L., Dickinson, J. M., Walker, D. K., ... Rasmussen, B. B. (2011). Skeletal muscle amino acid transporter expression is increased in young and older adults following resistance exercise. Fournal of Applied Physiology, 111(1), 135-142.
Fealy, C. E., Mulya, A., Lai, N., \& Kirwan, J. P. (2014). Exercise training decreases activation of the mitochondrial fission protein dynamin-related protein-1 in insulin-resistant human skeletal muscle. Fournal of Applied Physiology, 117(3), 239-245.

Filadi, R., Greotti, E., Turacchio, G., Luini, A., Pozzan, T., \& Pizzo, P. (2015). Mitofusin 2 ablation increases endoplasmic reticulum mitochondria coupling. Proceedings of the National Academy of Sciences of the USA, 112(17), E2174-E2181.

Fratta Pasini, A. M., Stranieri, C., Rigoni, A. M., De Marchi, S., Peserico, D., Mozzini, C., ... Garbin, U. (2018). Physical exercise Reduces Cytotoxicity and Up-Regulates Nrf2 and UPR expression in Circulating cells of peripheral Artery disease Patients: An Hypoxic adaptation? fournal of Atherosclerosis and Thrombosis, 25(9), 808-820.

Gavilán, M. P., Vela, J., Castaño, A., Ramos, B., del Río, J. C., Vitorica, J., \& Ruano, D. (2006). Cellular environment facilitates protein accumulation in aged rat hippocampus. Neurobiology of Aging, 27(7), 973-982.

Gearhart, Jr., R. F., Lagally, K. M., Riechman, S. E., Andrews, R. D., \& Robertson, R. J. (2009). Strength tracking using the OMNI resistance exercise scale in older men and women. Fournal of Strength and Conditioning Research, 23(3), 1011-1015.

Grootjans, J., Kaser, A., Kaufman, R. J., \& Blumberg, R. S. (2016). The unfolded protein response in immunity and inflammation. Nature Reviezus Immunology, 16(8), 469-484.

Kang, E. B., Kwon, I. S., Koo, J. H., Kim, E. J., Kim, C. H., Lee, J., ... Cho, J Y. (2013). Treadmill exercise represses neuronal cell death and inflammation during A $\beta$-induced ER stress by regulating unfolded protein response in aged presenilin 2 mutant mice. Apoptosis: An International fournal on Programmed Cell Death, 18(11), 1332-1347.

Kitaoka, Y., Ogasawara, R., Tamura, Y., Fujita, S., \& Hatta, H. (2015). Effect of electrical stimulation-induced resistance exercise on mitochondrial fission and fusion proteins in rat skeletal muscle. Applied Physiology, Nutrition, and Metabolism, 40(11), $1137-1142$.

López-Otín, C., Blasco, M. A., Partridge, L., Serrano, M., \& Kroemer, G. (2013). The hallmarks of aging. Cell, 153(6), 1194-1217.

Mejías-Peña, Y., Estébanez, B., Rodriguez-Miguelez, P., Fernandez-Gonzalo, R., Almar, M., de Paz, J. A., ... Cuevas, M. J. (2016a). Impact of resistance training on the autophagyinflammation-apoptosis crosstalk in elderly subjects. Aging (Albany NY), 9(2), 408-418.

Mejías-Peña, Y., Rodriguez-Miguelez, P., Fernandez-Gonzalo, R., Martínez-Flórez, S., Almar, M., de Paz, J. A., ... GonzálezGallego, J. (2016b). Effects of aerobic training on markers of autophagy in the elderly. Age (Dordr), 38(2), 33.

Memme, J. M., Oliveira, A. N., \& Hood, D. A. (2016). Chronology of UPR activation in skeletal muscle adaptations to chronic contractile activity. American fournal of PhysiologyCell Physiology, 310(11), C1024-C1036.

Mihailidou, C., Chatzistamou, I., Papavassiliou, A. G., \& Kiaris, H. (2017). Modulation of pancreatic islets' function and survival during aging involves the differential regulation of endoplasmic reticulum stress by $\mathrm{p} 21$ and CHOP. Antioxidants \& Redox Signal, 27(4), 185-200.

Moreira, O., Estébanez, B., Martínez-Flórez, S., de Paz, J. A., Cuevas, M. J., \& González-Gallego, J. (2017). Mitochondrial function and mitophagy in the elderly: Effects of exercise. Oxidative Medicine and Cellular Longevity, 2017, 1.

Naidoo, N., Davis, J. G., Zhu, J., Yabumoto, M., Singletary, K., Brown, M., ... Baur, J. A. (2014). Aging and sleep deprivation induce the unfolded protein response in the pancreas: Implications for metabolism. Aging Cell, 13(1), 131-141.

Nakka, V. P., Prakash-Babu, P., \& Vemuganti, R. (2016). Crosstalk between endoplasmic reticulum stress, oxidative 
stress, and autophagy: Potential therapeutic targets for acute CNS injuries. Molecular Neurobiology, 53(1), 532-544.

Ogborn, D. I., McKay, B. R., Crane, J. D., Parise, G., \& Tarnopolsky, M. A. (2014). The unfolded protein response is triggered following a single, unaccustomed resistance-exercise bout. American fournal of Physiology-Regulatory, Integrative and Comparative Physiology, 307(6), R664-R669.

Pereira, B. C., da Rocha, A. L., Pinto, A. P., Pauli, J. R., de Souza, C. T., Cintra, D. E., ... da Silva, A. S. (2016). Excessive eccentric exercise-induced overtraining model leads to endoplasmic reticulum stress in mice skeletal muscles. Life Sciences, $145,144-151$.

Pinto, A. P., da Rocha, A. L., Pereira, B. C., Oliveira, L. D., Morais, G. P., Moura, L. P., et al. (2017). Excessive training is associated with endoplasmic reticulum stress but not apoptosis in the hypothalamus of mice. Applied Physiology, Nutrition, and Metabolism, 42(4), 354-360.

Rodriguez-Miguelez, P., Fernandez-Gonzalo, R., Almar, M., Mejías, Y., Rivas, A., de Paz, J. A., ... González-Gallego, J. (2014). Role of Toll-like receptor 2 and 4 signaling pathways on the inflammatory response to resistance training in elderly subjects. Age (Dordr), 36(6), 9734.

Rodriguez-Miguelez, P., Fernandez-Gonzalo, R., Collado, P. S., Almar, M., Martinez-Florez, S., de Paz, J. A., ... Cuevas, M. J. (2015). Whole-body vibration improves the anti-inflammatory status in elderly subjects through toll-like receptor 2 and 4 signaling pathways. Mechanisms of Ageing and Development, 150, 12-19.

Senft, D., \& Ronai, Z. A. (2015). UPR, autophagy, and mitochondria crosstalk underlies the ER stress response. Trends in Biochemical Sciences, 40(3), 141-148.

Smiles, W. J., Hawley, J. A., \& Camera, D. M. (2016). Effects of skeletal muscle energy availability on protein turnover responses to exercise. The fournal of Experimental Biology, 219( $\mathrm{Pt} 2), 214$ 225.
Soto-Pantoja, D. R., Wilson, A. S., Clear, K. Y., Westwood, B., Triozzi, P. L., \& Cook, K. L. (2017). Unfolded protein response signaling impacts macrophage polarity to modulate breast cancer cell clearance and melanoma immune checkpoint therapy responsiveness. Oncotarget, 8(46), 80545-80559.

Takeda, N., Kume, S., Tanaka, Y., Morita, Y., Chin-Kanasaki, M., Araki, H., ... Uzu, T. (2013). Altered unfolded protein response is implicated in the age-related exacerbation of proteinuria-induced proximal tubular cell damage. The American Fournal of Pathology, 183(3), 774-785.

Tampakakis, E., Tabit, C. E., Holbrook, M., Linder, E. A., Berk, B. D., Frame, A. A., ... Hamburg, N. M. (2016). Intravenous lipid infusion induces endoplasmic reticulum stress in endothelial cells and blood mononuclear cells of healthy adults. Fournal of the American Heart Association, 5(1), e002574.

Tamura, Y., Matsunaga, Y., Kitaoka, Y., \& Hatta, H. (2017). Effects of heat stress treatment on age-dependent unfolded protein response in different types of skeletal muscle. The fournals of Gerontology. Series A, Biological Sciences and Medical Sciences, 72(3), 299-308.

Wu, H., Wei, H., Sehgal, S. A., Liu, L., \& Chen, Q. (2016). Mitophagy receptors sense stress signals and couple mitochondrial dynamic machinery for mitochondrial quality control. Free Radical Biology \& Medicine, 100, 199-209.

Wu, J., Ruas, J. L., Estall, J. L., Rasbach, K. A., Choi, J. H., Ye, L., .. Spiegelman, B. M. (2011). The unfolded protein response mediates adaptation to exercise in skeletal muscle through a PGC-1 $\alpha /$ ATF6 $\alpha$ complex. Cell Metabolism, 13(2), 160-169.

Zampieri, S., Pietrangelo, L., Loefler, S., Fruhmann, H., Vogelauer, M., Burggraf, S., ... Kern, H. (2015). Lifelong physical exercise delays age-associated skeletal muscle decline. The Fournals of Gerontology. Series A, Biological Sciences and Medical Sciences, 70(2), 163-173.

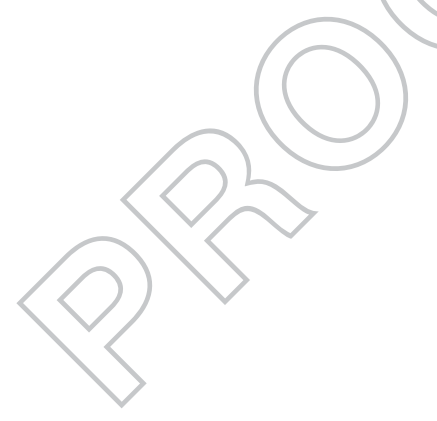

